\title{
Effect of COVID-19 Pandemic on HIV/AIDS, Tuberculosis, Malaria, and Maternal and Child Health Services, Uganda, 2020
}

\section{Daniel Jacob Emong ( $\sim$ demong@musph.ac.ug )}

Uganda Public Health Fellowship Program Ignatius Wadunde

Uganda Public Health Fellowship Program

Daniel Kadobera

Uganda Public Health Fellowship Program

Lilian Bulage

Uganda Public Health Fellowship Program

Steven Ndugwa Kabwama

Uganda Public Health Fellowship Program

Alex Riolexus Ario

Uganda Public Health Fellowship Program

\section{Research Article}

Keywords: COVID-19, HIV/AIDS, Tuberculosis, Malaria, Maternal and Child Health, Uganda

Posted Date: January 27th, 2022

DOI: https://doi.org/10.21203/rs.3.rs-1137771/v1

License: (1) This work is licensed under a Creative Commons Attribution 4.0 International License.

Read Full License 


\section{Abstract}

Background: Access to healthcare services has been severely compromised in many countries by the COVID-19 pandemic. By June 30, 2020 Uganda had not gathered enough information on the impact of COVID-19 on healthcare services. We assessed the effect of COVID-19 pandemic on human immunodeficiency virus (HIV), Tuberculosis (TB), Malaria, and Maternal and Child Health (MCH) services.

Methods: We conducted a cross-sectional assessment during July 2020 in randomly selected health facilities across the four geographical regions of Uganda. We abstracted data on the number of clients seeking healthcare services from, the Out-Patient Department (OPD), HIV, TB, malaria, and MCH facility registers before (January-February) and during (April-May) the pandemic. We compared the outcomes before and during using the paired t-test.

Results: We assessed 28 facilities. There was a significant decrease in average outpatient department total attendances from $(M=5,529, S D=6541.2)$ before compared to $(M=3576, S D=4345.6)$ during COVID19 pandemic $(P=0.0031)$. Similarly, the average number screened for TB significantly decreased from $(M=2822, S D=5409.2)$ to $(M=1300, S D=2525.5) P=0.040$ and $A N C 1^{\text {st }}$ contacts from $(M=200 S D=207.8)$ before to $(M=137, S D=133.3)$ during COVID-19 pandemic $p=0.015$.

Conclusion: A significant number of the population may not have readily accessed healthcare services during the pandemic especially those with long term illnesses like TB and HIV. We developed Information, Education and Communication materials and conducted a catch-up campaign for TB. Awareness creation among service providers on reduced intake of HIV and $\mathrm{MCH}$ services during COVID-pandemic is needed for enhancement of community education.

\section{Introduction}

Access to health services can be severely compromised during public health emergencies [1] such as coronavirus disease 2019 (COVID-19) which has challenged healthcare systems around the world due to increase demand for care. [2]. Despite the increasing demand for care of COVID-19 patients, healthcareseeking for conditions such as HIV/AIDS, tuberculosis, malaria and, maternal and child health (MCH) remains critical. The maintenance of healthcare services during the COVID-19 pandemic is particularly important for the most vulnerable populations, such as children, older persons, people living with chronic conditions, minorities, and people living with disabilities [3].

Understanding healthcare-seeking and ensuring continuity amidst the COVID-19 pandemic involves assessing the number of those turning up at health facilities for health care and implementing interventions that strengthen seeking of routine and essential health services [2]. When those seeking routine and essential health services receive timely medical care, it can buffer against detrimental stages of the disease and minimize transmissions in the community [4]. The World Health Organization (WHO) recommends countries understand the extent of disruptions across all healthcare services, the levels and reasons for disruptions and, the mitigation strategies to maintain essential health service delivery [5]. 
In Uganda health services are provided by the Ministry of Health (MoH), Ministry of Local Government (MoLG), and private and non-governmental organizations (NGOs) [6]. Ministry of Health is responsible for planning and developing health policies and for providing healthcare in all government hospitals while the MoLG is in charge of healthcare delivery at the district and lower levels [7].

The SARS-CoV-2 virus that causes COVID-19 was first detected in Uganda on March 21, 2020. By June $18,2020,741$ cases had been identified, most of them imported from incoming cargo truck drivers from neighbouring countries or airport travellers and their contacts [8]. On March 18, 2020, a national lockdown was declared, in response to the COVID-19 pandemic, and concern of the spread of cases without aggressive measures to prevent spread. A total lockdown was implemented on 30 March 2020 in Uganda that lasted until 26 May 2020 [9].

However, a month after the first case was detected, Uganda had not assessed the levels of disruptions $f$ health services for implementation of interventions to ensure consistent access to high-quality essential health services during the pandemic.. We assessed the effect of the COVID-19 pandemic on healthcareseeking for HIV/AIDS, Tuberculosis (TB), Malaria, and Maternal and Child Health (MCH) services to inform immediate planning and action.

\section{Methods}

\section{Study design}

We conducted a cross-sectional assessment in July 2020 in selected health facilities across the four geographical regions of Uganda. To estimate the effect of the COVID-19 pandemic on HIV/AIDS, TB, Malaria, and $\mathrm{MCH}$ services, we abstracted data on clients who accessed healthcare before and during the pandemic. We defined "before" as the first two months (January and February 2020) before March 2020, when the first case was detected and "during" as the first two months (April and May) after March 2020.

\section{Sample size considerations and sampling procedure}

We randomly selected two districts in each of the four geographical regions; eastern, northern, central, and western regions with at least one COVID-19 Treatment Unit (CTU). We randomly identified 32 health facilities and considered 28 with available facility registers at the time of this study. We purposefully considered the Regional Referral Hospital (RRH) and one Private Not for Profit (PNFP) with the highest Out-Patients Department (OPD) daily attendance in the selected district and randomly selected a health centre III and health centre II from the lists provided by the district health officer. The selected health facilities were representative of the different levels of health service delivery in the districts.

We considered all Out Patients Department (OPD), HIV/AIDS, TB, malaria and, Maternal and Child health clients registered in the health facility registers before and during the pandemic. 


\section{Study variables and data collection}

To assess the effect of the COVID-19 pandemic on the number of HIV/AIDS, TB, Malaria and, Maternal and Child Health clients seeking health services before and during the COVID-19 pandemic, we extracted data variables on the number of clients seeking healthcare services from all possible points of contacts in the health facility including OPD total attendances, number screened for TB, number of presumptive TB cases identified from OPD, number of cases diagnosed with TB, total new and relapsed TB cases registered in TB treatment unit, number of contacts of TB patients started on TPT, number of Specimen Referred, average Turn Around Time (Days) and number of pending results/ feedback. For malaria, the study variables included suspected fevers, total Malaria, Malaria confirmed (B/S and RDT), and Malaria cases treated. For Maternal and Child health, we extracted data on number of admissions, live birth, newborn deaths (0-7days), neonatal Death 8-28 days, maternal Death, postnatal Attendances, ANC 1st contacts, ANC No. in 1st Trimester and total ANC contacts. And for HIV, we abstracted data on HIV+ Mothers newly enrolled in $\mathrm{MCH}$ groups, HIV+ pregnant and women initiated on ART for EMTCT at any visit irrespective of when tested HIV+.

\section{Data Analysis}

We used paired t-test to determine statistically significant differences in the means before and during the COVID-19 pandemic for all the numerical data. We considered statistically significant at $5 \%$ level of significance

\section{Results}

Number of clients seeking tuberculosis services at health facilities before and during COVID-19, Uganda, 2020

There were total of 28 health facilities visited during this study. The health facilities that offered to screen TB were 25(89.3\%). There was a significant decrease in average OPD total attendances, number clients screened for TB, presumptive TB cases identified from OPD, total new and, relapsed TB cases registered in TB treatment unit during COVID-19 pandemic (Table 1). 
Table 1

Comparison of clients seeking tuberculosis services at health facilities before and during COVID-19, Uganda, 2020

\begin{tabular}{|c|c|c|c|c|c|}
\hline \multirow[t]{2}{*}{ Variables } & \multicolumn{2}{|c|}{ Before COVID-19 } & \multicolumn{2}{|c|}{ During COVID-19 } & \multirow[t]{2}{*}{ P-Value } \\
\hline & Mean $(\mathrm{N} / 28)$ & SD & Mean $(\mathrm{N} / 28)$ & SD & \\
\hline OPD total attendances & 5529 & 6541.2 & 3576 & 4345.6 & $0.0031^{*}$ \\
\hline Screened for TB & 2822 & 5409.2 & 1300 & 2525.5 & $0.0401^{*}$ \\
\hline OPD presumptive TB cases & 68 & 157.3 & 26 & 57.8 & $0.0380^{*}$ \\
\hline Cases diagnosed with TB & 8 & 17.4 & 4 & 9.0 & 0.0833 \\
\hline Total new and relapsed TB & 15 & 26.9 & 9 & 16.2 & $0.0384^{*}$ \\
\hline Patients started on TPT & 3 & 8.9 & 1 & 1.5 & 0.1708 \\
\hline Specimen referred & 6 & 12.0 & 74 & 309.2 & 0.3500 \\
\hline Average turnaround time (days) & 3 & 6.3 & 2 & 5.9 & 0.2934 \\
\hline Pending results/ feedback & 1 & 1.8 & 1 & 2.7 & 0.4895 \\
\hline \multicolumn{6}{|c|}{ *statistically significant at $5 \%$ level of significance } \\
\hline \multicolumn{6}{|c|}{${ }^{*} N$ - total number of exiting respondents from all the 28 health facilities } \\
\hline \multicolumn{6}{|l|}{$\star S D-S t a n d a r d$ Deviation } \\
\hline$\star M-M e a n$ & & & & & \\
\hline
\end{tabular}

Number of clients seeking malaria services at health facilities before and during COVID-19, Uganda, 2020

There was no significant difference in the average number of suspected fevers total malaria cases, malaria confirmed cases and malaria treated before and during the Covid-19 pandemic (Table 2).

Table 2

Comparison of clients seeking Malaria services at health facilities before and during COVID-19, Uganda, 2020

\begin{tabular}{|llllll|}
\hline \multirow{2}{*}{ Variables } & \multicolumn{2}{l}{ Before COVID-19 } & \multicolumn{2}{l}{ During COVID-19 } & \multirow{2}{*}{ P-Value } \\
\cline { 2 - 5 } & Mean (N/28) & SD & Mean (N/28) & SD & \\
\hline Suspected fevers & 952 & 880.8 & 736 & 552.6 & 0.2179 \\
\hline Total malaria & 610 & 504.9 & 478 & 448.1 & 0.0532 \\
\hline Malaria confirmed (B/S and RDT) & 502 & 416.4 & 442 & 444.1 & 0.3707 \\
\hline Malaria cases treated & 479 & 427.0 & 443 & 446.1 & 0.6593 \\
\hline
\end{tabular}


Number of clients seeking maternal and child health services at health facilities before and during COVID19, Uganda, 2020

There was a significant decrease in the average number of ANC first contacts reported by the health facilities during COVID-19 137 (SD=133.3) than before the COVID-19 epidemic 200 (SD=207.8); $p=0.015$. Total ANC contacts reported by the health facilities decreased significantly during the COVID-19 epidemic. (Table 3)

Table 3

Comparison of clients seeking maternal and child -antenatal care services at health facilities before and during COVID-19, Uganda, 2020

\begin{tabular}{|c|c|c|c|c|c|}
\hline \multirow[t]{2}{*}{ Variables } & \multicolumn{2}{|c|}{ Before COVID-19 } & \multicolumn{2}{|c|}{ During COVID-19 } & \multirow[t]{2}{*}{ P-Value } \\
\hline & $\begin{array}{l}\text { Mean } \\
(\mathrm{N} / 28)\end{array}$ & SD & $\begin{array}{l}\text { Mean } \\
(\mathrm{N} / 28)\end{array}$ & SD & \\
\hline ANC 1st contacts & 200 & 207.8 & 137 & 133.3 & $0.0155^{\star}$ \\
\hline ANC No. in 1 st Trimester & 50 & 46.8 & 52 & 59.0 & 0.7982 \\
\hline Total ANC contacts & 713 & 728.2 & 499 & 493.2 & $0.0198^{*}$ \\
\hline
\end{tabular}

Maternal and child health of Obstetric and newborn care services before and during Covid-19 showed that the average number of admissions to health facilities significantly reduced from 323 before to 233 during COVID-19 $(P=0.005)$. Live births at health facilities also reduced from 258 before to 188 during COVID-19 $(p=0.0077)$ (Table 4). 
Table 4

Comparison of average number of clients seeking maternal and child healthObstetric and newborn care services before and during COVID-19, Uganda, 2020

\begin{tabular}{|c|c|c|c|}
\hline \multirow[t]{2}{*}{ Variables } & Before COVID-19 & During COVID-19 & \multirow[t]{2}{*}{ P-Value } \\
\hline & Mean (N/28) & Mean (N/28) & \\
\hline Number of admissions & 323 & 233 & $0.0050 *$ \\
\hline Live birth & 258 & 188 & $0.0077^{*}$ \\
\hline New-born deaths (0-7days) & 5 & 4 & 0.1852 \\
\hline Neonatal Death 8-28 days & 1 & 1 & 0.3910 \\
\hline Maternal Death & 1 & 1 & 0.8255 \\
\hline Postnatal Attendances & 291 & 237 & 0.0594 \\
\hline
\end{tabular}

\section{Number of clients seeking Prevention of Mother-To-Child Transmission of HIV}

There was no significant decrease in the average number of HIV+ Mothers newly enrolled in $\mathrm{MCH}$ groups by health facilities during the COVID-19 epidemic $1(S D=2.4) ; p=0.326$ compared to before the COVID-19 epidemic $M=2(S D=3.4)$

Similarly, there was no significant difference in the number of HIV+ pregnant women initiated on ART for eMTCT at any visit irrespective of when tested HIV+ by health facilities during the COVID-19 pandemic 4 $(S D=8.7) ; p=0.564$ compared to before the COVID-19 pandemic $5(S D=8.1)($ Table 5$)$

Table 5

Comparison of clients seeking HIV-PMTCT services before and during Covid-19

\begin{tabular}{|c|c|c|c|c|c|}
\hline \multirow[t]{2}{*}{ Variables } & \multicolumn{2}{|c|}{$\begin{array}{l}\text { Before COVID- } \\
19\end{array}$} & \multicolumn{2}{|c|}{$\begin{array}{l}\text { During COVID- } \\
19\end{array}$} & \multirow[t]{2}{*}{$\begin{array}{l}\text { P- } \\
\text { Value }\end{array}$} \\
\hline & $\begin{array}{l}\text { Mean } \\
(\mathrm{N} / 28)\end{array}$ & SD & $\begin{array}{l}\text { Mean } \\
(\mathrm{N} / 28)\end{array}$ & SD & \\
\hline $\mathrm{HIV+}$ Mothers newly enrolled in $\mathrm{MCH}$ groups & 2 & 3.4 & 1 & 2.4 & 0.326 \\
\hline HIV+ pregnant & 5 & 8.1 & 4 & 8.7 & 0.564 \\
\hline $\begin{array}{l}\text { women initiated on ART for eMTCT at Any visit } \\
\text { irrespective of when tested HIV+ }\end{array}$ & & & & & \\
\hline
\end{tabular}

\section{Discussion}


This study revealed an overall decline in the number of patients seeking healthcare services during the covid-19 pandemic. The overt difference in average number of patients seeking healthcare before and during COVID-19, could largely attributed to the widespread covid-19 interventions such as transport restrictions, curfew, diversion of health personnel and resources to respond to the COVID-19 pandemic. The reduction might also be owed to the barrier posed by patients' fears of contracting Covid-19 [10]. In addition, many healthcare seekers could have perceived negative reception at the facility during COVID-19 by the health professionals as reported in Ghana [10]. They noted that healthcare professionals were applying unfriendly and high-level infection prevention protocols especially to patients presenting with cough and flu symptoms which affected care seeking among the population.

The monthly notification average data of number screened for TB, the number of presumptive TB cases identified from OPD, and the number of cases diagnosed with TB sharply declined during the covid-19 outbreak (April-May) compared to the monthly notification average data before covid-19 (JanuaryFebruary). The travel restrictions during the lock down might have made it difficult for presumptive TB patients to visit health facilities and seek medical care. The presumptive TB tracing and referral could have also been affected by the reallocation of health workers to fight the COVID-19 pandemic.

In terms of sputum examination and other laboratory diagnosis for TB, there was a clear slump in the number of patients seeking laboratory services during COVID-19. These could be because of increasing number of covid-19 cases that may have overwhelmed the Uganda healthcare system and necessitated transfer of TB laboratory to COVID-19 testing and temporarily closed their TB outpatient clinic during the intensive period of COVID-19, leaving TB patients with nowhere to go for sputum examination [11]. This may impact TB treatment and diagnostic services for those seeking TB healthcare services [12].

Our study showed that the number of malaria diagnoses did not significantly change during the COVID19 outbreak (April-May), although many studies have indicated that disruptions to malaria control activities have resulted in a resurgence in malaria morbidity and mortality. The minimal decrease in the number of malaria cases diagnosed could have been attributed to shortage of medication suppliers with VHTs that subsequently resulted into the continuous referrals of patients by Village health teams (VHTs) to health facilities for Artemisinin-based combination therapies (ACTs) and laboratory diagnosis of malaria at health facilities. VHTs implement Integrated Community Case Management (iCCM) of malaria, diarrhoea and pneumonia [13]. The VHTs who previously got close to sick people in the community, could, have run short of ACT supply or feared contracting COVID-19 and preferred referral[14]. However, while Malaria elimination campaigns must reach marginalized groups distant from the health facilities, the programmes are at risk of being scaled back for logistic reasons associated with COVID-19 interventions, putting communities at risk [15].

The data from our study also shows that ANC 1st contacts at the health facilities were severely affected by COVID - 19. Like our findings, health facilities in Rwanda saw a decline in antenatal attendances in the first months of the pandemic [16]. Reduction of mortality and morbidities from vaccine-preventable diseases in developing countries involves successfully implementing strategies that ensure high 
coverage and minimize drop-outs and missed opportunities [17] [18]. The decreases in childhood vaccinations could have detrimental vaccine-preventable disease outbreaks in the future due to COVID-19 disruptions of health seeking. The reasons for disrupted services could be attributed to fear of being exposed to people with COVID-19 at health facilities.

This study showed no difference in the numbers of mothers receiving PMTCT services at health facilities before and during the COVID-19 pandemic. The Joint United Nations Programmes on HIV/AIDS (UNAIDS) equally reported that the impact on services for the prevention of vertical transmission of HIV (from mother to child) is mixed-by April, some countries generally saw a decline in the number of women tested for HIV at their first antenatal clinic visit [19], but by June 2020 that decline had been reversed as corroborated with our findings [20]. PMTCT services are received during Antenatal appointments. It is important for pregnant mothers to attend antenatal appointments, as these are the times when they can get an HIV test, receive treatment if tested HIV positive, and medical advice to help keep them and their babies healthy [21]. On the other hand, this study revealed severe decline in the expected live births, obstetric and newborn care clients seeking healthcare at health facilities during the COVID-19 pandemic. This could be because most mothers resorted to the community traditional birth attendants (TBAs) as was the case in Kenya [22]. In Kenya, most mothers resorted to giving birth in the homes of the traditional birth attendants, since most health facilities were temporarily shut down and health workers were reassigned to the COVID-19 crisis. These results underline the additional stressors of the COVID-19 pandemic overlaid on already stretched obstetric and newborn care services, like in Nepal [23], While the government of Uganda actively discourages TBA-supported births preferring that TBAs refer mothers to the nearest health facility, it would be better to the integrate traditional birth attendants during pandemics such as COVID-19 and other crises.

Focus on pandemic management and response with limited oversight of providing care to those suffering from other illness, healthcare personnel being required to quarantine, or becoming ill or dying, and therefore not being available for routine services. All these factors may have contributed to unwillingness to seek for healthcare and therefore cause delays in the diagnosis and commencement of treatment.

\section{Limitations}

Our findings should be interpreted in line with the following limitations. We used health facility registers data as captured by the facility Health Management Information System (HMIS) focal persons who were not on duty in some facility during the study period. Some clients seeking healthcare during COVID-19 pandemic could have not been captured in the registers because of the HMIS focal persons absence for fear of contracting COVID-19 infection. So, our magnitude of those seeking health services during COVID_19 pandemic was likely an underestimate. Also, during the pandemic, several categories of triages were set up at facilities for different categories of clients seeking healthcare services and it is most likely that some were not captured in the OPD register. The clients who sought healthcare services before 
COVID-19 freely interreacted with the facility HMIS focal persons and followed the one health facility triage were captured in all the relevant registers, hence less likely to be underestimated.

\section{Conclusion}

A significant number of the population may not have readily accessed health services during the pandemic lockdown especially those with long term illness like TB and HIV. We developed Information, Education and Communication materials (IEC) and conducted a three-month catch-up campaign for TB. We recommended that the Ministry of Health $(\mathrm{MOH})$ should reintroduce community outreaches with clear SOPs to bridge the challenge of seeking healthcare during pandemic. For example, strengthening community refill models for long term illness patients, registering, monitoring, and linking all antenatal mothers to the health facilities through the VHTs. We further recommended that the Ministry of Health $(\mathrm{MOH})$ sensitizes the population to continuously seek healthcare services and to allow people with long term illness to routinely access treatment during the pandemic from any nearby health facilities without referral letters.

\section{Abbreviations}

AIDS: Acquired Immune-Deficiency Syndrome

ANC: Antenatal care

Cl: Confidence interval;

COVID-19: Coronavirus Disease;

DHIS2: District Health Information system version 2

EMTCT: Elimination of Mother to Child Transmission of HIV, first antenatal care visit, first antenatal care visit

HIV: Human Immunodeficiency Virus

HMIS: Health Management Information System

MCH: Maternal and Child Health

MoH: Ministry of Health

MTCT: Mother-to-Child Transmission of HIV PITC: Provider Initiated Testing and Counseling

PMTCT: Prevention of Mother to Child Transmission of HIV;

PPE: Personal protective equipment; 
SARS-CoV-2: Severe Acute Respiratory Syndrome Coronavirus 2;

SD: Standard Deviation

\section{Declarations}

\section{Ethics approval}

This assessment was conducted as part of the Ministry of Health and Makerere University School of Public Health efforts to respond to the COVID-19 pandemic and associated impact. All methods were carried out in accordance with the U.S. Centers for Disease Control and Prevention relevant guidelines and regulation. We obtained ethical approval from the Higher Degrees Research and Ethics Committee of Makerere University School of Public Health. Permission to use the survey data was obtained from Makerere University School of Public Health, the Uganda Public Health Fellowship Program where the survey is housed and the Uganda Centers for Disease Control and Prevention (CDC). Participants provided written informed consent to participate in this study and were informed of study benefits and risks before agreeing to participate. Participants were free to withdraw from the study without losing the benefits arising from the study. We obtained permission to extract the data from patient registers from health facilities' administrators with the consent of the district health officers. The data extracted was regarded private and confidential. Profile details of data subjects were not captured. Individual data were coded with numeric identifiers instead of participant names to ensure confidentiality. In effort to protect the research assistants and healthcare workers in the study health facilities, the COVID-19 prevention measures of wearing face mask, physically/socially distancing, and using hand sanitizers were ensured.

\section{Consent for publication}

Not applicable

\section{Availability of data and materials}

The datasets upon which our findings are based belong to the Uganda Public Health Fellowship Program. For confidentiality reasons the datasets are not publicly available. However, the data sets can be availed upon reasonable request from the corresponding author and with permission from the Uganda Public Health Fellowship Program

\section{Competing interest}

The authors declare they have no competing interest.

\section{Funding and disclaimer}

This project was supported by the President's Emergency Plan for AIDS Relief (PEPFAR) through the US Centers for Disease Control and Prevention Cooperative Agreement number GH001353-01 through Makerere University School of Public Health to the Uganda Public Health Fellowship Program, MoH. Its 
contents are solely the responsibility of the authors and do not necessarily represent the official views of the US Centers for Disease Control and Prevention, the Department of Health and Human Services, Makerere University School of Public Health, or the MoH. The staff of the funding body provided technical guidance in the design of the study, ethical clearance and collection, analysis, and interpretation of data and in writing the manuscript

\section{Authors' contributions}

D.J.E took the lead in conceptualizing the study design, data collection, and analysis assisted by I.W under the supervision of L.B., D. K, S.N.K and A.R.A All authors participated substantially in the writing, read, and approved the final manuscript for publication. L.B, DK, and A.R.A had primary responsibility for final content.

\section{Acknowledgments}

We thank the healthcare workers who participated in this study. We are also grateful to the support from the administration of Mulago National Referral Hospital, Entebbe Grade B Hospital, Arua Regional Referral Hospital, Jinja Regional Referral Hospital, and Kabale Regional Referral Hospital for their support in conducting this investigation.

\section{References}

1. M. M. Ruprecht et al., "Evidence of Social and Structural COVID-19 Disparities by Sexual Orientation, Gender Identity, and Race/Ethnicity in an Urban Environment," J. Urban Heal., 2021, doi: 10.1007/s11524-020-00497-9.

2. J. Bigio and P. Madhukar, "How Covid is making it tougher to tackle TB, AIDS, malaria and child health," The Print, 2020.

3. S. Bhandari et al., "The sequel to COVID-19: the antithesis to life," J. Ideas Heal., 2020, doi: 10.47108/jidhealth.vol3.issspecial1.69.

4. L. A. O. Ngo Bibaa, "Primary health care beyond COVID-19: dealing with the pandemic in Cameroon," BJGP Open, 2020, doi: 10.3399/bjgpopen20X101113.

5. D. Wise, C. Walsh, T. Chanin, R. Downey, T. Parr, and S. Mercer, “PG85 Up-skilling the workforce. Preparing to return to frontline medicine in the support of COVID-19," 2020, doi: 10.1136/bmjstel2020-aspihconf.133.

6. M. Nabukeera, "Challenges and Barriers to the Health Service Delivery System in Uganda," J. Nurs. Heal. Sci., 2016.

7. D. K. Henriksson, S. S. Peterson, P. Waiswa, and M. Fredriksson, "Decision-making in district health planning in Uganda: Does use of district-specific evidence matter?," Heal. Res. Policy Syst., 2019, doi: 10.1186/s12961-019-0458-6. 
8. R. Galiwango, J. Kitayimbwa, A. N. Kiragga, K. E. Atkins, A. L. Brown, and A. K. Mbonye, "Modelling the Impact and Public Health Response to COVID-19 in Uganda," SSRN Electron. J., 2020, doi: $10.2139 /$ ssrn.3633199.

9. R. Namazzi, R. Opoka, A. L. Conroy, D. Datta, M. Goings, and C. C. John, "Impact of a National Lockdown for COVID-19 on Morbidity and Mortality Among Children with Sickle Cell Anaemia at a Tertiary Care Hospital in Uganda," Blood, 2020, doi: 10.1182/blood-2020-142417.

10. F. I. Saah, H. Amu, A. A. Seidu, and L. E. Bain, "Health knowledge and care seeking behaviour in resource-limited settings amidst the COVID-19 pandemic: A qualitative study in Ghana," PLoS One, 2021, doi: 10.1371/journal.pone.0250940.

11. H. Fei et al., "The impact of the COVID-19 epidemic on tuberculosis control in China," Lancet Reg. Heal. - West. Pacific, 2020, doi: 10.1016/j.lanwpc.2020.100032.

12. T. Togun, B. Kampmann, N. G. Stoker, and M. Lipman, "Anticipating the impact of the COVID-19 pandemic on TB patients and TB control programmes," Annals of Clinical Microbiology and Antimicrobials. 2020, doi: 10.1186/s12941-020-00363-1.

13. D. Mubiru et al., "Evaluation of integrated community case management in eight districts of Central Uganda," PLoS One, 2015, doi: 10.1371/journal.pone.0134767.

14. K. McKague, "South Sudanese refugees in Uganda face overwhelming odds against COVID-19 (Special Issue: Coronavirus pandemic)," South Sudan Med. J., 2020.

15. World Health Organization (WHO), "Community-based health care, including outreach and campaigns, in the context of the COVID-19 pandemic," Who. 2020.

16. J. Burt et al., "Indirect Effects of COVID-19 on Maternal, Neonatal, Child, Sexual and Reproductive Health Services in Kampala, Uganda," medRxiv, 2021.

17. A. Zewdie, M. Letebo, and T. Mekonnen, "Reasons for defaulting from childhood immunization program: A qualitative study from Hadiya zone, Southern Ethiopia," BMC Public Health, 2016, doi: 10.1186/s12889-016-3904-1.

18. D. N. Shikuku et al., "Door - To - door immunization strategy for improving access and utilization of immunization Services in Hard-to-Reach Areas: A case of Migori County, Kenya," BMC Public Health, 2019, doi: 10.1186/s12889-019-7415-8.

19. A. Goga et al., "Centring adolescent girls and young women in the HIV and COVID-19 responses," The Lancet. 2020, doi: 10.1016/S0140-6736(20)32552-6.

20. UNAIDS, "COVID-19's impact on HIV vertical transmission - UNAIDS," 2020. https://www.unaids.org/en/resources/presscentre/featurestories/2020/october/20201027_COVID19-impact-hiv-vertical-transmission (accessed Oct. 27, 2020).

21. E. Du Plessis et al., "Prevention of mother-to-child transmission of HIV in Kenya: Challenges to implementation," BMC Health Serv. Res., 2014, doi: 10.1186/1472-6963-14-S1-S10.

22. S. O. Ombere, "Access to Maternal Health Services During the COVID-19 Pandemic: Experiences of Indigent Mothers and Health Care Providers in Kilifi County, Kenya," Front. Sociol., 2021, doi: $10.3389 /$ fsoc. 2021.613042 . 
23. A. KC et al., "Effect of the COVID-19 pandemic response on intrapartum care, stillbirth, and neonatal mortality outcomes in Nepal: a prospective observational study," Lancet Glob. Heal., 2020, doi: 10.1016/S2214-109X(20)30345-4. 